\title{
A Semantic Model to Assist Policy Refinement Mechanisms for NFV-MANO Systems
}

\author{
Michel Bonfim ${ }^{1}$, Fred Freitas ${ }^{1}$, Stênio Fernandes ${ }^{1}$ \\ ${ }^{1}$ Centro de Informática (CIn) - Universidade Federal de Pernambuco (UFPE) \\ Recife - PE - Brazil \\ \{msb6,fred, sflf\}@cin.ufpe.br
}

\begin{abstract}
Management in NFV scenarios is a complex task. In this scenario, automated policy refinement can be used to enforce NFV-MANO functions to deal with the increased complexity. However, existing solutions do not perform policy analysis. Therefore, in this work, we propose a semantic model in OWL 2, named Onto-Planner, to assist the policy refinement process for NFV-MANO systems. Preliminary results show that Onto-Planner provides policy analysis when a DL reasoner is applied.
\end{abstract}

\section{Introduction}

Management in Network Function Virtualization (NFV) scenarios is a complex task since delivering such Virtual Network Functions (VNFs) and Network Services (NSs) over the physical infrastructure requires flexible and adaptable NFV Management and Orchestration (NFV-MANO) systems [ETSI 2014]. The increasing complexity of the NFV-MANO has widened the gap between human intention and the managed system behavior. Therefore, new solutions are necessary to reduce this gap and improve these complex systems' management.

In this context, Policy-based Management (PBM) systems can be used to enforce NFV-MANO functions as a way to deal with the increased complexity. PBM systems are the key enablers to provide flexibility and adaptability in NFV-MANO systems. Assisted by policies, NFV-MANO functions can be provided in an automated fashion, aiming to meet the dynamic requirements of Network Service Orchestration (NSO) and Resource Orchestration (RO). An NFV-PBM system refers to the management of rules governing the behavior of NFV-MANO. However, PBM is not a straightforward task [Riekstin et al. 2016]. This situation is exacerbated when considering its application in the management of NFV systems since NFV-MANO must provide several functionalities in a flexible and adaptable way. In this scenario, a key issue regarding PBM systems arises, namely, policy refinement.

Policy Refinement is the process of transforming high-level policies (Goals or Intents), which are not directly executable in a management system, into directly enforceable, low-level policies (Event-Condition-Action (ECA) rules). In most systems, policy refinement is done manually. Automated policy refinement is a nontrivial process, and it remains a much-neglected research area. It has been severely dismissed due to its inherent complexity [Machado et al. 2017].

According to [Riekstin et al. 2016], one of the key issues for automated policy refinement is Policy Analysis. A refinement solution must ensure that the refined specification achieves the requirements and is consistent with system properties and limitations, 
as well as with existing policies [Craven et al. 2010]. Since disputes occur among a set of policies, pairwise detection will not suffice [Bouten et al. 2016]. To perform policy analysis, a semantic model (ontology) to describe the goals and low-level rules may be created. Such ontology would be expressed in Web Ontology Language (OWL) Description Logic (DL) [Krötzsch et al. 2012], which - besides the well-defined, non-ambiguous semantics above mentioned for the vocabulary being defined - provides decidable, sound and complete inference for some reasoning tasks, such as inconsistency checking.

Aiming at moving forward on the Network Management Research Group (NMRG) standardization threads, this work proposes the use of a semantic model in OWL 2 [Krötzsch et al. 2012] to assist the policy refinement process for NFV-MANO systems, called Onto-Planner. In summary, our main contributions are as follows:

1. A language for describing goals in NFV environments;

2. The Onto-Planner ontology, which offers a vocabulary with its complex relations and constraints of the domain, expressive enough to describe different types of alarms, goals, and ECA rules that will be used in the refinement process;

3. The Onto-Planner axioms and Semantic Web Rule Language (SWRL) ${ }^{1}$ rules that provides policy analysis, when a DL reasoner is applied.

\section{Network Function Virtualization (NFV)}

NFV is transforming the computer and communication networks industry. Rather than dealing with proprietary hardware equipment, which includes a limited set of specific network applications, NFV allows users to transfer network functions from specific to Common Off-The-Shelf (COTS) hardware using virtualization technologies [ETSI 2014]. These network functions are then called VNFs and perform different network operations: Firewall, Network Address Translation (NAT), Deep Packet Inspection (DPI), Load Balancing, among others.

NFV aims at creating NSs that interconnect one or more VNFs to support the creation and deployment of end-to-end network services [ETSI 2014]. Some benefits of deploying network services as virtual functions are: (i) flexibility in the allocation of network functions in general-purpose hardware; rapid implementation and deployment of new network services; support of multiple versions of service and multi-tenancy scenarios; reduction in Capital Expenditure (CAPEX) costs by managing energy usage efficiently; and automation of the operational processes, thus improving efficiency and reducing Operational Expenditure (OPEX) costs.

The NFV architecture comprises three main functional blocks: VNF, NFV Infrastructure (NFVI), and NFV-MANO. VNF is the virtualization of a specific network function, which should operate independently of the others. A particular VNF can also be divided into several sub-functions called VNF Components (VNFCs), where there is one Virtual Machine (VM) implementing each VNFC. On the other hand, NFVI comprises all hardware and software required to deploy, operate, and monitor VNFs. To this end, NFVI has a virtualization layer necessary for abstracting the hardware resources (processing, storage, and network connectivity). Finally, NFV-MANO performs both NSO and RO. Such functionalities include the following non-exhaustive list of operations [ETSI 2014]:

\footnotetext{
${ }^{1}$ https://www.w3.org/Submission/SWRL/
} 
VNF and NS lifecycle management, VNF and NS scaling, resource orchestration, and management in multi-domain scenarios, access control, and performance and fault management.

For these purposes, NFV-MANO comprises three components.

- The Virtualized Infrastructure Manager (VIM), which manages and controls the interaction of VNFs with physical resources under its control (e.g., allocation, deallocation, and inventory);

- The VNF Manager (VNFM), which is responsible for managing the VNF lifecycle (e.g., initialization, suspension, and termination); and

- The NFV Orchestrator (NFVO), which is responsible for realizing NS on NFVI. It also performs NFVI monitoring as a way to collect information for operations and performance management.

\section{Related Work}

In NFV systems, we can identify two types of policies: application-specific and management-specific. The former consists of domain-specific policies that can be set for both a single NS and globally for all services such as network function precedence, location constraints, and resource usage. The latter consists of the rules that will be generated by the refinement process and stored in NFV-PBM to govern system behavior. We only found works that perform policy analysis to detect conflicts between application-specific policies [Bouten et al. 2016, Bonfim et al. 2019], and one of them is our previous work [Bonfim et al. 2019]. Therefore, we argue that Onto-Planner is the first semantic model that perform policy analysis to detect conflicts between management-specific policies.

We found only two works that provide a solution for NFV policy refinement. [Scheid et al. 2017] proposed an Intent-Based Networking (IBN) solution, named INSpIRE. This solution rely on a Non-Functional Requirement (NFR) framework to perform the refinement procedure. [Jacobs et al. 2019] proposed an intent definition language, called Nile, to fill the gap between human intention and the network configurations. Nilebased code is compiled to generate the lower-level configuration commands.

It is worth mentioning that none of the above works perform policy analysis. They did not take into account the effects of actions/policies have over the managed objects (system state) during the refinement process. In this context, Onto-Planner can be adapted to support such solutions to avoid conflicts between different intents and lowlevel policies.

\section{Onto-Planner}

To compose Onto-Planner classes, we reused two information models: NOVI Policy from the NOVI (Network Innovation over Virtualized Infrastructures) Information $\mathrm{Model}^{2}$, and Policy Core Information Model (PCIM) ${ }^{3}$. It is worth noting that we reused them to define policy rules and their different parts in a vendor-independent manner.

Onto-Planner enables the description of various objects that make up the state of a policy refinement domain for NFV. With Onto-Planner at hand, an operator or

\footnotetext{
${ }^{2}$ https://www.sciencedirect.com/science/article/abs/pii/S0167739X13002811

${ }^{3}$ https://tools.ietf.org/html/rfc3060
} 
NFV-MANO may record different types of alarms and goals that will be used in the refinement process. Besides, at the end of this process, Onto-Planner may be used to describe the generated enforceable policies (ECA rules). Finally, conflicts between these elements (alarms, goals, ECA rules) are carried out through DL inconsistency verification when a DL semantic reasoner is relied upon.

We organized the Onto-Planner classes into two categories: classes used before and after the refinement process. The first category encompasses all classes used for the following descriptions:

- NFV-MANO operators: deals with the described NFV-MANO elements that are manageable (e.g., NFVO) and the operators that allow them to be managed, defined according to European Telecommunications Standards Institute (ETSI) specifications. The following classes are used for this description: ManagedEntity and ManagedEntityMethod;

- Alarms: deals with the description of different types of alarms. To describe an alarm, the operator (via Threshold API) should specify one of the predefined metrics (instances of the Metric class) for the alarm to watch, define the threshold (Threshold class) for that metric, and the type of action (from a predefined list - Action class) to perform when the alarm is triggered. Onto-Planner currently allows alarm creation for auto-healing and auto-scaling. Further details will be presented in Section 4.1;

- Goals: deals with the description of different types of high-level goals. An individual of the Goal class indicates a goal's existence. Besides, a single goal must be associated with a single NS (NS class), one or more VNFs (VNF class) belonging to that NS, a single level (Level class), and at least one service attribute (Attribute class). Further details are presented in Section 4.2;

- Events and Parameters: deal with the description of possible events (Event class) and parameters (Parameter class) that can be used to compose the events and conditions, respectively, of the generated enforceable policies. Further details are in Section 4.3.

It is worth noting that all of the above descriptions are used in the refinement process.

The second category comprises all classes used after the refinement process for the description of enforceable policies, and subsequent conflict detection and diagnosis. There are classes that help the description of ECA rules such as PolicyRule, PolicyEvent, PolicyCondition, and PolicyAction. Furthermore, there are classes that assist an ECA rule to support alarm creation into the NFV-PBM. Since Onto-Planner only supports healing and scaling alarms, we create only the following classes: HealingGroup, HealingPolicy, HealingCriteria, ScalingGroup, ScalingPolicy, and ScalingCriteria. They offer a way of describing enforceable alarms that is supported by different MANO frameworks such as Open Source MANO ${ }^{4}$. Such classes only describe the alarms selected during refinement. Further details about enforceable policies description are in Section 4.3.

Additionally, Onto-Planner has all sibling classes disjoint because they describe different elements of the domain.

\footnotetext{
${ }^{4}$ https://osm.etsi.org/
} 


\subsection{Describing Alarms}

Multiple NFV-MANO frameworks such as Open Baton ${ }^{5}$ and Open Source MANO ${ }^{6}$ support fault management. Such feature enables the creation and execution of alarms in the form of ECA Rules to deal with possible problems that may occur in NFVI. Alarms help attend to the requirement of dynamicity regarding current or future system state and topology changes. Therefore, in addition to enforceable policies that govern NFV-PBM behavior, we must also generate rules that include alarms in our refinement process.

Onto-Planner enables the operator to record different alarm types for the refinement process. In this context, one operator can specify the metric for the alarm to watch, the threshold for that metric, and the kind of action (from a predefined list) to be executed when the alarm fires. To this end, one prerequisite must be met: all available metrics must be previously recorded as Metric individuals. Such metrics should be accessible by the target NFV-MANO. The ontology maintainer should carry out this task, i.e., someone responsible for editing Onto-Planner (via, e.g., Protégé tool ${ }^{7}$ ).

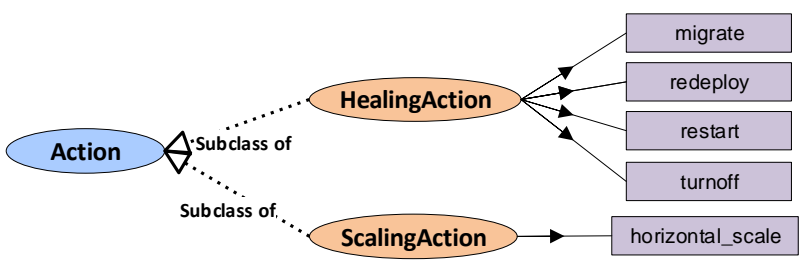

(a) Action subclasses.

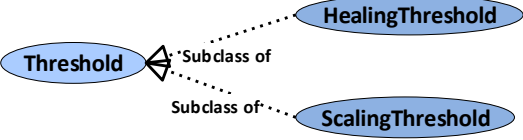

(b) Threshold subclasses.

Figure 1. Healing and Scaling subclasses.

Onto-Planner currently allows alarm creation for auto-healing and auto-scaling. Figure 1 shows the classes and subclasses available for these purposes. The Action class enables the description of all actions (class individuals) necessary to alarm creation (see Figure 1a). To group these operations, we created two defined classes [Krötzsch et al. 2012], subclasses of Action. The HealingAction class encompasses only healing-oriented actions. It is currently limited to the following individuals: $\mathbf{m i}$ grate, redeploy, restart, turnoff. These individuals represent common healing actions [ETSI 2016]. The ScalingAction class includes only scaling-oriented actions. It is currently limited to only one individual: horizontal_scale. Horizontal scaling means adding or removing virtualized computer resources into/from your pool of resources [ETSI 2015]. Finally, it is possible to define non-functional parameters for Action individuals such as its subject and target.

The Threshold class allows description of custom thresholds for both healing and scaling actions. For this, it has two subclasses: HealingThreshold and ScalingThreshold (see Figure 1b). Figure 2a shows how a healing alarm can be described. An individual of type HealingAction must have a relationship of type hasThreshold with an individual of type HealingThreshold. In its turn, the HealingThreshold individual must have three

\footnotetext{
${ }^{5}$ Open Baton website: https://openbaton.github.io/

${ }^{6}$ Open Source MANO website: https://osm.etsi.org/

${ }^{7}$ Protégé website: https://protege.stanford.edu/
} 


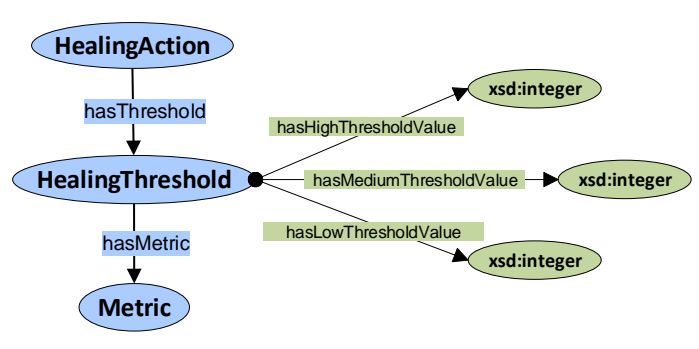

(a) Healing alarms.

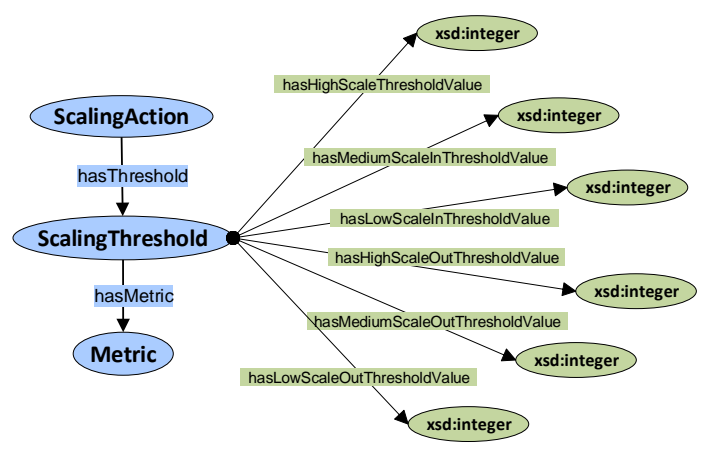

(b) Scaling alarms.

Figure 2. Describing alarms.

data properties set with integer values. These data properties define thresholds for the three possible levels of a goal: low, medium, and high. Besides, the HealingThreshold individual must have a relationship of type hasMetric with an individual of type Metric.

Similarly, one can describe a scaling action, as shown in Figure 2b. A ScalingThreshold individual type must have six data properties set instead of three. The new data property is because horizontal scaling has two possible operations: scale in (i.e., removing resources) and out (i.e., adding resources). Therefore, we must define thresholds for both operations.

Finally, some restrictions (axioms) have been defined in the above classes to limit the scope in creating alarms. In fact, a Threshold element must be associated with at least one Metric. Furthermore, a Threshold element must have exactly one data property set for each level and each operation in case of ScalingThreshold.

\subsection{Describing Goals}

According to [Han and Lei 2012], the use of goals (or intents) allows the specification of the desired states instead of a sequence of actions. In this context, we can declare the high-level goals, such as Service Level Agreements (SLAs), and hence generate enforceable policies to govern NFV-MANO behavioral choices while satisfying the goals. Besides, goal-oriented refinement assists in reducing both Capital Expenditures (CAPEX) and Operational Expenditures (OPEX).

In this context, Onto-Planner enables NFVO to record goal policies extracted from Network Service Descriptor (NSD). It deals with the description of different types of high-level goals. To this end, we proposed the following language to define high-level goals in a NS Request (NS-Req) (Listing 1):

\section{Listing 1. Goal Language Grammar}

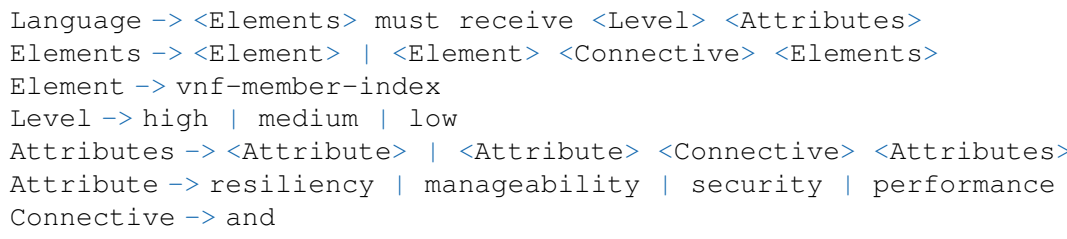


A tenant can define one or more goals in the same NS-Req. Each goal has three (3) parts:

- Elements: specifies which NS-specific VNFs the goal should be applied to. To identify a VNF, we set the parameter vnf-member-index, which indicates the position of the VNF in the service chain;

- Level: determines the goal's degree of importance, i.e., how critical this goal is for the NS (high, medium or low);

- Attributes: defines one or more service attributes for the goal. According to ETSI [ETSI 2014], the overall NS attributes are reliability, availability, manageability, security and performance. For the grammar, we replaced reliability and availability by resilience since ETSI considers that resiliency is an aspect of QoS that can be characterized by the combination of reliability and availability [ETSI 2015]. ing 2):

For a better understanding, let us consider the following goal policy example (List-

\section{Listing 2. Goal Example}

1 and 2 must receive high performance and resilience

This goal establishes that NFV-MANO must provide high performance and resilience for the VNFs in positions 1 and 2 within the NS.

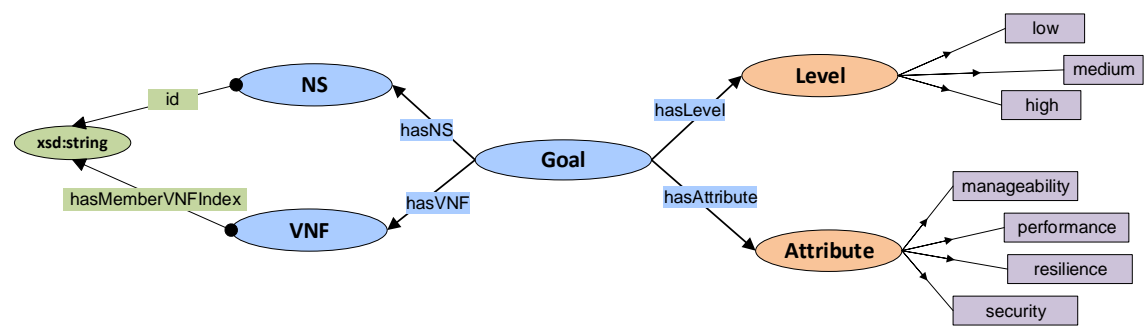

Figure 3. Describing high-level goals.

According to Figure 3, a Goal individual must be associated with a single NS (NS class), one or more VNFs (VNF class) belonging to that NS, a single level (Level class), and at least one service attribute (Attribute class). Table 1 lists the axioms that delimit these restrictions.

Both Level and Attribute classes are defined classes [Krötzsch et al. 2012]. The Level class is limited to the following individuals: low, medium, high. They determine the degree of importance of the goal, i.e., how critical this goal is for the NS. In its turn, the Attribute class is restricted to the following individuals: resiliency, manageability, security, and performance, as defined by ETSI [ETSI 2014].

\subsection{Describing Policy Rules}

Once a Policy Refinement Mechanism executes, it generates a set of enforceable policies (ECA rules) and alarms for a previously registered goal policy. Those elements can be described in the Onto-Planner for inconsistency checking (policy analysis). In this section, we show how to describe ECA rules and enforceable alarms. 
Table 1. Class Axioms for Goal description (Manchester Syntax).

\begin{tabular}{|l|}
\hline Goal SubClassOf hasNS max $1 \mathrm{NS}$ \\
\hline Goal SubClassOf hasVNF some VNF \\
\hline Goal SubClassOf hasLevel max 1 Level \\
\hline Goal SubClassOf hasAttribute some Attribute \\
\hline
\end{tabular}

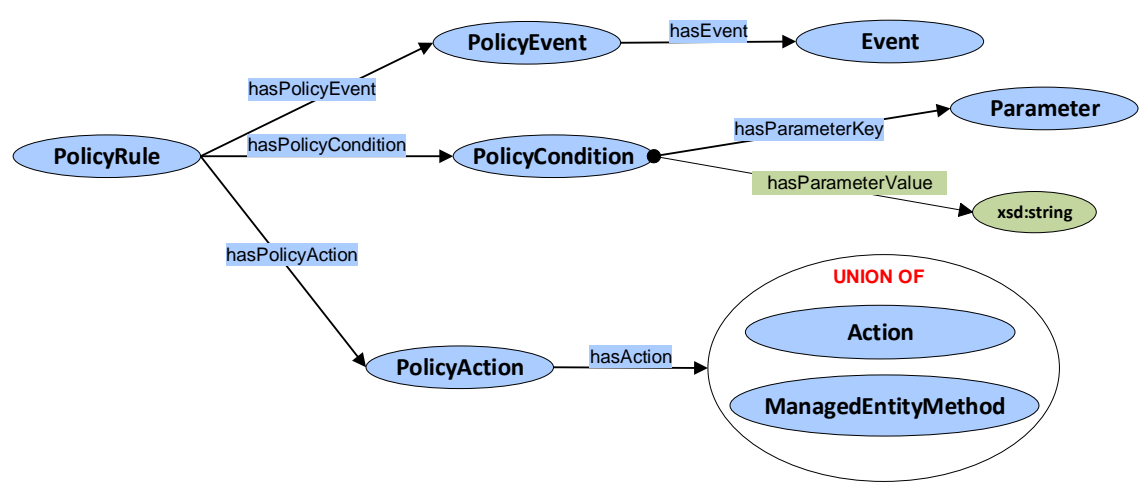

Figure 4. Describing low-level policy rules.

As shown in Figure 4, we define the following classes to describe ECA rules: PolicyRule, PolicyEvent, PolicyCondition, and PolicyAction. A PolicyRule individual must have relationship with exacly one PolicyEvent, via the hasPolicyEvent object property, and exacly one PolicyAction, via hasPolicyAction object property. Besides, a PolicyRule must have one or more PolicyCondtion elements; this relationship is defined by the hasPolicyCondition object property.

Furthermore, an individual of the PolicyEvent class must be associated (hasEvent property) with one or more Event individuals. The Event class deals with the description of all existing events in an NFV-MANO such as "network service instantiated" or "VNF instance scaled". It is noteworthy that possessing multiple hasEvent relationships in the same PolicyEvent indicates that all events must occur to trigger the rule.

Table 2. Class Axioms for ECA Rule description (Manchester Syntax).

\begin{tabular}{|l|}
\hline PolicyRule SubClassOf hasPolicyAction exactly 1 PolicyAction \\
\hline PolicyRule SubClassOf hasPolicyEvent exactly 1 PolicyEvent \\
\hline PolicyEvent SubClassOf hasEvent some Event \\
\hline PolicyAction SubClassOf hasAction some (Action or ManagedEntityMethod) \\
\hline PolicyCondition SubClassOf hasParamenterKey exactly 1 Parameter \\
\hline
\end{tabular}

In addition, one PolicyAction may be related (hasAction property) with one or more actions, which may be of type Action or ManagedEntityMethod. Finally, each PolicyCondition must be associated (hasParameterKey property) with only one parameter type (Parameter class), which can include multiple string values (hasParameterValue data property). The Parameter class deals with the description of all existing parameters in an NFV-MANO such as "network service id" or "alarm type". Table 2 lists the created axioms that reflect the above restrictions. 


\subsection{Managing Policy Conflicts}

Onto-Planner enables the following conflict checks: Alarm Conflicts, Goal Conflicts, and ECA Rule Conflicts.

Concerning Alarm Conflicts, we want to detect if there are at least two alarms with the same metric and the same action, an obvious policy conflict. For this, we create the object property usedBySameActionAs, relating two Metric individuals, that are used by alarms that apply the same action.

Nevertheless, we still have a critical challenge: the definition of which statements or propositions reflect the behavior of this function. For the property to reflect its expected behavior, it has to Irreflexive. For instance, let $x$ and $y$ be individuals of the Metric class. If $x$ is used by the same action as $y$, then $x$ cannot be $y$.

Besides, we specify an SWRL rule in (1) to generate implicit facts with the usedBySameActionAs object property: if we have an action $x$ that has two different thresholds $y$ and $z$, where $y$ has the metric $m 1$ and $z$ has the metric $m 2$, then there is a relationship of type usedBySameActionAs between these two metrics.

$$
\begin{array}{r}
\text { differentFrom }(y, z) \wedge \operatorname{Action}(x) \\
\wedge \text { hasThreshold }(x, z) \wedge \text { hasThreshold }(x, y) \\
\wedge \text { hasMetric }(y, m 1) \wedge \text { hasMetric }(z, m 2) \\
\rightarrow \text { usedBySameActionAs }(m 1, m 2)
\end{array}
$$

Concerning Goal Conflicts, we want to detect an intersection between one or more goals. Remarkably, intersections between goals can lead to duplicate policies at the end of the refinement process. Therefore, we should check this whenever a new goal is created. In this work, we consider two goals intersect when they share the same NS, one or more VNFs, and one or more attributes. Hence, again we create the intersects With object property to reflect this behavior. It relates two individuals of Goal class. Besides, we specify the SWRL rule in (2) to generate implicit facts with this object property.

$$
\begin{array}{r}
\operatorname{Goal}(g 1) \wedge \operatorname{Goal}(g 2) \wedge \text { differentFrom }(g 1, g 2) \\
\wedge h a s N S(g 1, n s 1) \wedge h a s N S(g 2, n s 2) \wedge i d(n s 1, i d t) \wedge i d(n s 2, i d t) \\
\wedge h a s V N F(g 1, v n f 1) \wedge h a s V N F(g 2, \text { vnf } 2) \\
\wedge \text { hasMemberVNFIndex }(\text { vnf } 1, \text { index }) \\
\wedge \text { hasMemberVNFIndex }(\text { vnf } 2, \text { index }) \\
\wedge \text { Attribute }(a) \wedge \text { hasAttribute }(g 1, a) \wedge \text { hasAttribute }(g 2, a) \\
\rightarrow \text { intersectsWith }(g 1, g 2)
\end{array}
$$

Suppose we have two goals $x$ and $y$. Once we execute the DL reasoner, the following facts will be inferred: intersectsWith $(x, y)$ and intersectsWith $(y, x)$.

However, these facts alone are insufficient to generate inconsistency in ontology, which is the approach used to detect conflicts. The object property intersectsWith has to be Asymmetric to meet this constraint. For example, let $x$ and $y$ be individuals of the Goal class. If $x$ intersects with $y$, then $y$ cannot intersects with $x$. By applying this restriction, Onto-Planner is inconsistent whenever the rule in (2) infers facts with the intersectsWith object property.

Concerning ECA Rule Conflicts, both alarm and goal conflict detection are performed whenever one of these elements is created in the ontology. This cautionary ap- 
proach seeks to prevent enforceable policies from being generated with conflicts after the refinement process. Nonetheless, there is still a kind of conflict to avoid: the duties' conflict. According to [Bandara et al. 2003], this conflict arises if the same subject performs a task that encompasses operations that, in the context of the application, are defined to be conflicting, for example, instantiating and terminating the same VNF in the same task.

To solve this issue, we need to detect if at least two PolicyRule individuals share the same events and conditions but include conflicting actions. To make this case, we created the conflicts With object property to indicate that two actions are conflicting. This property links two individuals belonging to the set formed by the union of ManagedEntityMethod and Action classes. Before the refinement process, the ontology maintainer must define all conflicting actions, i.e., define facts with this object property. The ontology maintainer is then responsible for editing Onto-Planner without using the Protégé tool ${ }^{8}$, for example.

Besides, we specify the SWRL rule in (3) to generate implicit facts with the conflictsWith object property.

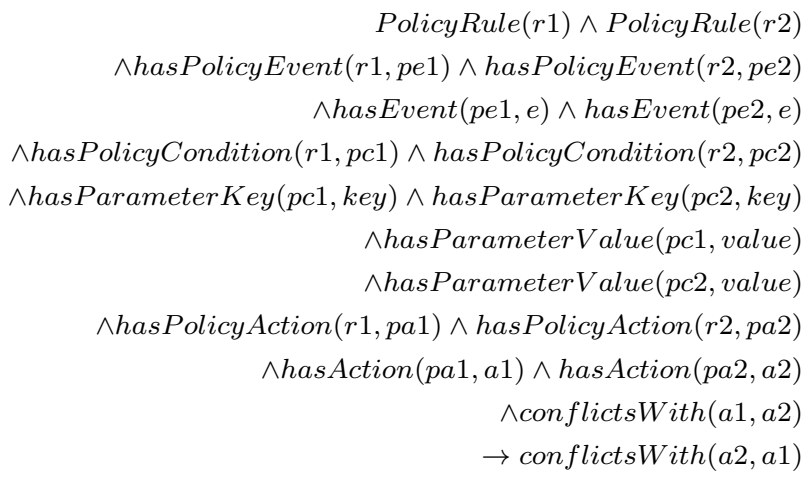

Suppose the following fact was previously created into Onto-Planner: conflictsWith(intantiate_vnf, terminate_vnf).

Now, suppose that, after the refining process, two PolicyRule individuals were generated: $x$ and $y$. These rules share the same events and conditions, but $x$ includes on instantiate_vnf action and $y$, a terminate_vnf action. Thus, the DL reasoner infers the following fact: conflictsWith(terminate_vnf, intantiate_vnf).

Analogously to other definitions above, conflitsWith is Asymmetric, so as to provoke an inconsistency. For example, let $x$ and $y$ be Action individuals. If $x$ intersects with $y$, then $y$ cannot intersects with $x$. Using this restriction, Onto-Planner will be inconsistent whenever the rule in (3) summon facts with the conflitsWith object property.

We emphasize that, unfortunately, we have not been able to define axioms using role chains. According to OWL $2.0 \mathrm{GRs}^{9}$, once we set an object property as irreflexive or asymmetric, we can only define simple object property expressions for it, and thus, property chains are prohibitive. This is the rationale behind the above SWRL rules employing property chains.

\footnotetext{
${ }^{8}$ Protégé website: https://protege.stanford.edu/

${ }^{9}$ OWL 2.0 GR website: http: / /www.w3.org/TR/owl2-syntax/
} 


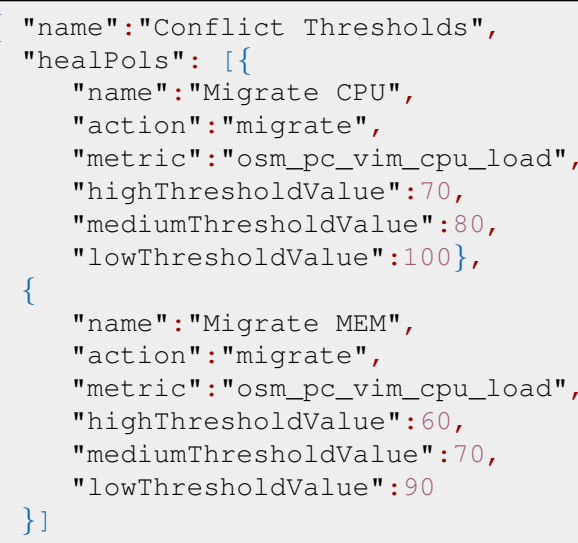

(a) JSON request Use Case 1.

Figure 5. Detecting and Diagnosing conflicts between alarms.

\section{Onto-Planner Validation}

In this section, we present the process and results of Onto-NFV validation. The process was carried out in two phases.

In the first phase, we implement Onto-Planner using the Protégé tool ${ }^{10}$. The source code is available in the following link: https://github.com/michelsb/atom/ blob/master/database/onto-planner. owl. Besides, we implement a Javabased RESTful API to manage Alarm, Goal and ECA rule individuals, relying on Spring Boot $^{11}$ and OWL API ${ }^{12}$. To create or remove those individuals, we use JSON as a data model. For conflict detection and diagnostic, we use the HermiT ${ }^{13}$, a fully compliant OWL 2 Reasoner for inconsistency verification.

In the second phase, we simulate three use cases (UC) to evaluate the capacity of Onto-Planner to detect and diagnose conflicts between alarms, goals, and ECA rules. We describe them below.

\subsection{Use Case 1 - Testing Conflicts between Alarms}

As mentioned in Subsection 4.4, Onto-Planner allows the operator to verify if there are alarms in which the same metric applies twice for the same action. In this use case, we simulate conflict between two alarms by inserting the two alarms (see Figure 5a) into Onto-Planner.

We create two alarms that point to the same action (i.e., migrate) and the same metric (i.e., osm_pc_vim_cpu_load). In this case, when the reasoner is performed, it detects inconsistencies in the Onto-Planner and presents the explanations shown in Figure $5 b$.

Such explanations state that, since there is the SRWL rule (1), the reasoner infers the following relationships (line 6): usedBySameActionAs(osm_pc_vim_cpu_load,osm_pc_vim_cpu_load).

\footnotetext{
${ }^{10}$ Protégé website: https://protege.stanford.edu/

${ }^{11}$ Spring Boot website: https://spring.io/projects/spring-boot

${ }^{12}$ OWL API website: http://owlapi.sourceforge.net/

${ }^{13}$ HermiT website: http://www.hermit-reasoner.com/
} 


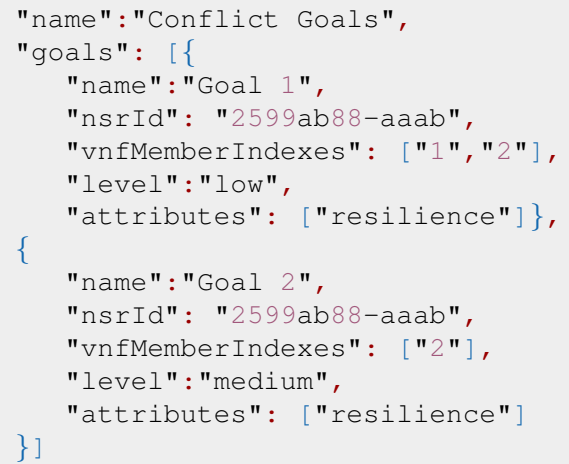

(a) JSON request Use Case 2.

\begin{tabular}{|l|}
\hline 1) goal-1 hasNS goal-1-ns-2599ab88-aaab \\
\hline 2) goal-2 hasNS goal-2-ns-2599ab88-aaab \\
\hline 3) goal-1-ns-2599ab88-aaab id “2599ab88-aaab” \\
\hline 4) goal-2-ns-2599ab88-aaab id "2599ab88-aaab” \\
\hline 5) goal-1 has VNF goal-1-vnf-2 \\
\hline 6) goal-2 hasVNF goal-2-vnf-2 \\
\hline 7) goal-1-vnf-2 hasMemberVNFIndex “2” \\
\hline 8) goal-2-vnf-2 hasMemberVNFIndex “2” \\
\hline 9) goal-1 hasAttribute resilience \\
\hline 10) goal-2 hasAttribute resilience \\
\hline 11) DifferentIndividuals: goal-1, goal-2 \\
\hline 12) SWRL Rule in (2) \\
\hline 13) AsymmetricObjectProperty: intersects With \\
\hline
\end{tabular}

(b) Explanation for the inconsistency detected in Use Case 2.

Figure 6. Detecting and Diagnosing conflicts between Goals.

However, this assertion cannot be valid since usedBySameActionAs is irreflexive (line 7), as mentioned in Subsection 4.4, thus generating inconsistencies.

\subsection{Use Case 2 - Testing Conflicts between Goals}

As mentioned in Subsection 4.4, Onto-Planner allows the NFVO to detect conflicts between goals that apply to the same NS and share the same attributes and VNFs. In this use case, we simulate such a conflict by inserting the two goals (see Figure 6a) into OntoPlanner.

In this scenario, we create two goals that point to the same NS with id " $2599 \mathrm{ab} 88$ aaab" and share the same VNFs (i.e., member index 2) and attributes (i.e., resilience). In this case, when the reasoner is performed, it detects inconsistencies in the Onto-Planner and presents the explanations shown in Figure $6 \mathrm{~b}$.

Such explanations state that, since there is the SRWL rule (2), the reasoner infers the following relationships (line 12): intersectsWith(goal-1, goal-2) and intersectsWith (goal-2, goal-1).

However, this assertion cannot be valid since intersectsWith is asymmetric (line 13), as mentioned in Subsection 4.4, and the individuals goal-1 and goal-2 are different (line 11), thus generating inconsistencies.

\subsection{Use Case 3 - Testing Conflicts between Rules}

As mentioned in Subsection 4.4, Onto-Planner allows the NFVO to detect conflict of duties. Such conflict will arise if the same subject should perform a task that encompasses operations that are explicitly defined to be conflicting in the context of the application. For example, instantiate and terminate the same VNF in the same task. Therefore, we simulate the detection and diagnosis of conflicts of duties in this use case.

To this end, first, we define a relationship conflictsWith between two previously created operations: create phy_nic_bonding and create_load_sharing_between_links.

Besides, we created two ECA rules. Both rules were described to be triggered by the same events and conditions. In addition, one has create phy_nic_bonding as action, 
while the other has create_load_sharing_between_links as action. Finally, once executed, the reasoner detects inconsistencies in the Onto-Planner and presents the explanations shown in Figure 7.

\begin{tabular}{|l|}
\hline $\begin{array}{l}\text { 1) create_phy_nic_bonding conflictsWith } \\
\text { create_load_sharing_between_links }\end{array}$ \\
\hline 2) pol_create_load_sharing_between_links Type PolicyRule \\
\hline 3) pol_create_phy_nic_bonding Type PolicyRule \\
\hline 4) pol_create_load_sharing_between_links hasPolicyEvent object26 \\
\hline 5) pol_create_load_sharing_between_links hasPolicyAction object25 \\
\hline 6) object25 hasAction create_load_sharing_between_links \\
\hline 7) pol_create_phy_nic_bonding hasPolicyAction object21 \\
\hline$\ldots$ \\
\hline 11) object21 hasAction create_phy_nic_bonding \\
\hline$\ldots$ \\
\hline 18) SWRL Rule in (3) \\
\hline 19) AsymmetricObjectProperty: conflictsWith \\
\hline
\end{tabular}

Figure 7. Explanation for the inconsistency detected in Use Case 3.

Such explanations state that, since there is a relationship conflictsWith between the operations create phy_nic_bonding and create_load_sharing_between_links (line 1), and since there are two ECA rules, named pol_create_load_sharing_between_links (line 2) and pol_create_phy_nic_bonding (line 3), that include those two operations (lines 6 and 11), the reasoner infers the following relationship from the SRWL rule (2) (line 18): conflictsWith(create_load_sharing_between_links, create_phy_nic_bonding)

However, this assertion cannot be valid since conflictsWith is asymmetric (line 19), as mentioned in Subsection 4.4, thus generating inconsistencies.

\section{Conclusion}

This paper proposed the Onto-Planner, a semantic model in OWL 2 that enables the description of different types of alarms and goals used in the refinement process. Besides, at the end of this process, Onto-Planner may be used to describe the generated ECA rules. Finally, when a DL semantic reasoner is relied upon, it performs policy analysis to detect conflicts between those elements. Preliminary experiments demonstrate that OntoPlanner can be used to assist the policy refinement process for NFV-MANO systems. However, it is worth mentioning that these experiments are only in their initial phase. In this case, for future work, it would be good to provide a performance evaluation.

It is noteworthy that the implementation of a Policy Refinement Mechanism and an NFV-PBM is not part of the scope of this work. Onto-Planner aims to assist and improve the operation of these solutions. An example of an NFV-PBM architecture can be found in [ETSI 2017].

Finally, taking into account the latest efforts made by IETF/IRTF, future activities include pursuing contributions to standardization along the way Onto-Planner can be used to support intelligent network management mechanisms (Intent-Based Networking) for NFV systems in an efficient and scalable way.

\section{References}

Bandara, A. K., Lupu, E. C., and Russo, A. (2003). Using event calculus to formalise policy specification and analysis. In Proceedings of the 4th IEEE International Workshop 
on Policies for Distributed Systems and Networks, POLICY '03, pages 26-, Washington, DC, USA. IEEE Computer Society.

Bonfim, M., Freitas, F., and Fernandes, S. (2019). A semantic-based policy analysis solution for the deployment of nfv services. IEEE Transactions on Network and Service Management, 16(3):1005-1018.

Bouten, N., Claeys, M., Mijumbi, R., Famaey, J., Latré, S., and Serrat, J. (2016). Semantic validation of affinity constrained service function chain requests. In 2016 IEEE NetSoft Conference and Workshops (NetSoft), pages 202-210.

Craven, R., Lobo, J., Lupu, E. C., Russo, A., and Sloman, M. (2010). Decomposition techniques for policy refinement. In Proceedings of the 6th International Conference on Network and Service Management, CNSM 2010, Niagara Falls, Canada, October 25-29, 2010, pages 72-79.

ETSI (2014). Network Functions Virtualisation (NFV) - Architectural Framework. ETSI GS NFV 002 V1.2.1.

ETSI (2015). Network Functions Virtualisation (NFV) - Resiliency Requirements. ETSI GS NFV-REL 001 V1.1.1.

ETSI (2016). Network Functions Virtualisation (NFV) - Reliability - Report on Models and Features for End-to-End Reliability. ETSI GS NFV-REL 003 V1.1.2 (2016-07).

ETSI (2017). Network functions virtualisation - management and orchestration - report on policy management in mano (release 3). ETSI GR NFV-IFA 023 V3.1.1.

Han, W. and Lei, C. (2012). A survey on policy languages in network and security management. Computer Networks, 56(1):477-489.

Jacobs, A. S., Pfitscher, R. J., Ferreira, R. A., and Granville, L. Z. (2019). Refining network intents for self-driving networks. SIGCOMM Comput. Commun. Rev., 48(5):5563.

Krötzsch, M., Patel-Schneider, P., Rudolph, S., Hitzler, P., and Parsia, B. (2012). OWL 2 web ontology language primer (second edition). Technical report, W3C. http://www.w3.org/TR/2012/REC-owl2-primer-20121211/.

Machado, C. C., Wickboldt, J. A., Granville, L. Z., and Filho, A. E. S. (2017). ARKHAM: an advanced refinement toolkit for handling service level agreements in softwaredefined networking. J. Network and Computer Applications, 90:1-16.

Riekstin, A. C., Januario, G. C., Rodrigues, B. B., Nascimento, V. T., de Brito Carvalho, T. C. M., and Meirosu, C. (2016). A survey of policy refinement methods as a support for sustainable networks. IEEE Communications Surveys and Tutorials, 18(1):222-235.

Scheid, E. J., Machado, C. C., Franco, M. F., dos Santos, R. L., Pfitscher, R. P., SchaefferFilho, A. E., and Granville, L. Z. (2017). Inspire: Integrated nfv-based intent refinement environment. In 2017 IFIP/IEEE Symposium on Integrated Network and Service Management (IM), pages 186-194. 